\title{
ALGEBRAIC K3 SURFACES WITH FINITE AUTOMORPHISM GROUPS
}

\author{
SHIGEYUKI KONDŌ
}

\section{Introduction}

The purpose of this paper is to give a proof to the result announced in [3]. Let $X$ be an algebraic surface defined over $C . \quad X$ is called a $K 3$ surface if its canonical line bundle $K_{X}$ is trivial and $\operatorname{dim} H^{1}\left(X, \mathcal{O}_{X}\right)=0$. It is known that the automorphism group $\operatorname{Aut}(X)$ of $X$ is isomorphic, up to a finite group, to the factor group $O\left(S_{X}\right) / W_{X}$, where $O\left(S_{X}\right)$ is the automorphism group of the Picard lattice of $X$ (i.e. $S_{X}$ is the Picard group of $X$ together with the intersection form) and $W_{X}$ is its subgroup generated by all reflections associated with elements with square $(-2)$ of $S_{X}$ ([11]). Recently Nikulin [8], [10] has completely classified the Picard lattices of algebraic $K 3$ surfaces with finite automorphism groups.

Our goal is to compute the automorphism groups of such $K 3$ surfaces. Let $X$ be an algebraic $K 3$ surface with finite automorphism group $\operatorname{Aut}(X)$. By definition, there exists a nowhere vanishing holomorphic 2-form $\omega_{X}$ on $X$. Since an automorphism $g$ of $X$ preserves $\omega_{X}$, up to constants, $g^{*} \omega_{X}=\alpha_{X}(g) \cdot \omega_{X}$ where $\alpha_{X}(g) \in C^{*}$. Therefore we have an exact sequence

$$
1 \longrightarrow G_{X} \longrightarrow \operatorname{Aut}(X) \stackrel{\alpha_{X}}{\longrightarrow} Z / m \longrightarrow 1
$$

where $Z / m$ is a cyclic group of $m$-th root of unity in $C^{*}$ and $G_{X}$ is the kernel of $\alpha_{X}$. Moreover the representation of the cyclic group $Z / m$ in $T_{X} \otimes Q$ is isomorphic to a direct sum of irreducible representations of the cyclic group $Z / m$ over $\boldsymbol{Q}$ of maximal rank $\phi(m)$, where $T_{X}$ is a transcendental lattice of $X$ and $\phi$ is the Euler function. In paticular $\phi(m) \leq$ rank $T_{X}$ and hence $m \leq 66$ ([6], Theorem 3.1 and Corollary 3.2).

An algebraic $K 3$ surface $X$ is called general if the image of $\alpha_{X}$ is of order at most 2 , and $X$ is called special if it is not general. The meaning of this definition is as follows: Let $X$ be an algebraic $K 3$ surface with

Received January 26, 1987. 
a Picard lattice $S_{X}$. Let $S$ be an abstract lattice which is isomorphic to $S_{X}$. Denote by $M_{S}$ the moduli space for algebraic $K 3$ surfaces whose Picard lattices are isomorphic to $S$. Then the dimension of $M_{S}$ is equal to $20-\operatorname{rank}(S)$. A general $K 3$ surface $Y$ with $S_{Y}=S$ corresponds to a point of the complement of hypersurfaces in $M_{S}$.

Theorem. Let $X$ be an algebraic $K 3$ surface with finite automorphism group $\operatorname{Aut}(X)$.

(i) If $X$ is general, then $\operatorname{Aut}(X)$ is as in the following table:

Table 1.

\begin{tabular}{c|c}
\hline \multicolumn{1}{c|}{$S_{X}$} & $\operatorname{Aut}(X)$ \\
\hline$U \oplus E_{8} \oplus E_{8} \oplus A_{1}$ & $\Im_{3} \times Z / 2$ \\
\hline$U \oplus E_{8} \oplus E_{8}, U \oplus E_{8} \oplus E_{7}$ & \\
$U \oplus E_{8} \oplus D_{6}, U \oplus E_{8} \oplus D_{4} \oplus A_{1}$ & \\
$U \oplus D_{8} \oplus D_{4}, U \oplus E_{8} \oplus A_{1}^{4}$ & \\
$U \oplus E_{7} \oplus A_{1}^{4}, U \oplus D_{6} \oplus A_{1}^{4}$ & $Z / 2 \times Z / 2$ \\
$U \oplus D_{4} \oplus A_{1}^{5}$ & \\
$U(2) \oplus D_{4} \oplus D_{4}, U \oplus A_{1}^{8}$ & \\
$U(2) \oplus A_{1}^{7}$ & $Z / 2$ or $\{1\}$ \\
\hline otherwise
\end{tabular}

where $U$ (resp. $U(2))$ is the lattice of rank 2 with the intersection matrix $\left(\begin{array}{ll}0 & 1 \\ 1 & 0\end{array}\right)\left(\right.$ resp. $\left.\left(\begin{array}{ll}0 & 2 \\ 2 & 0\end{array}\right)\right), A_{m}, D_{n}$ and $E_{k}$ are negative definite lattices associated with the Dynkin diagrams of type $A_{m}, D_{n}$ and $E_{k}$ respectively and $A_{1}^{k}$ denotes the direct sum $A_{1} \oplus A_{1} \oplus \cdots \oplus A_{1}$ ( $k$ times).

(ii) If $X$ is special, then $\operatorname{Aut}(X)$ is a cyclic extension of the group in the above table.

We remark here that there exists a special $K 3$ surface $X$ with $\operatorname{Aut}(X) \simeq Z / 66$. This automorphism acts on the Picard group of $X$ as identity. In [4], we studied automorphisms with this property.

Also for Enriques surfaces with finite automorphism groups, we refer the reader to [2], [9].

To prove the above theorem we use the following phenomenon: In 
the exact sequence (1), if $\operatorname{rank}\left(S_{X}\right)$ becomes smaller, then $G_{X}$ too becomes smaller, and the group $Z / m$ grows bigger.

In Section 1, we recall the Picard lattices of algebraic $K 3$ surfaces with finite automorphism groups. Section 2 is devoted to the results on finite automorphisms of $K 3$ surfaces due to Nikulin [6] and Mukai [5]. In particular from these results we obtain all the possible cases of $G_{X}$ (Lemma 2.3). In Sections 4 and 5 we prove the above theorem. In case $\operatorname{rank}\left(S_{X}\right) \geq 15$ we have the dual graph of all smooth rational curves on $X$ ([8], Sect. 4, Part 5, Table 2) and hence we can compute Aut $(X)$. In case $\operatorname{rank}\left(S_{X}\right) \leq 14$ it follows from the result in Section 2 that $G_{X}$ is a subgroup of $Z / 3$ or $Z / 2 \times Z / 2$. To determine Aut $(X)$ we use the theory of symmetric bilinear forms (cf. [7]) and that of elliptic pencils due to Kodaira [1] and Shioda [12] (Sect. 3).

\section{$\S 1$. Picard lattices of $K 3$ surfaces with finite automorphism groups}

In this section we recall the Nikulin's classification [8], [10] of Picard lattices of algebraic $K 3$ surfaces with finite automorphism groups.

A lattice $L$ is a free $Z$-module of finite rank endowed with an integral bilinear form $\langle$, $\rangle$. By $L_{1} \oplus L_{2}$ we denote the orthogonal direct sum of lattices $L_{1}$ and $L_{2}$. For a lattice $L$ and an integer $m$ we denote by $L(m)$ the lattice whose bilinear form is the one on $L$ multiplied by $m$. Also we denote by $U$ the lattice of rank 2 with the intersection matrix $\left(\begin{array}{ll}0 & 1 \\ 1 & 0\end{array}\right)$ and by $A_{m}, D_{n}$ and $E_{k}$ the negative definite lattices associated with the Dynkin diagram of type $A_{m}, D_{n}$ and $E_{k}$ respectively. A lattice $L$ is called even if $\langle x, x\rangle \in 2 Z$ for all $x \in L$. Let $S$ be a non degenerate lattice. We denote by $S^{*}=\operatorname{Hom}(S, Z)$ the dual of $S$. Put $A_{S}=S^{*} / S$. Then $A_{S}$ is a finite abelian group which is called the discriminant group of $S$. We denote by $l(S)$ the number of minimal generators of $A_{S}$. A lattice $S$ is called a 2-elementary if $A_{S}$ is a 2-elementary abelian group. For a 2elementary lattice $S$, we define a parity $\delta(S)$ of $S$ as follows:

$$
\delta(S)= \begin{cases}0 & \text { if } q_{S}(x)=0 \text { for all } x \in A_{S} \\ 1 & \text { otherwise }\end{cases}
$$

where $q_{S}$ is the quadratic form on $A_{S}$ induced from the one on $S$.

Proposition 1.1 ([8], Theorem 4.3.2). An indefinite 2-elementary even lattice is determined, up to isomorphisms, by the invariants ( $\operatorname{rank}(S), l(S)$, 
$\delta(S))$

The following tables give the description of Picard lattices of rank $\geq 9$ of algebraic $K 3$ surfaces with finite automorphism groups which we need for the proof of our theorem.

Table $2 \quad\left(S_{X}\right.$ is 2-elementary, rank $\left.S_{X} \geq 9\right)$.

\begin{tabular}{c|c}
\hline $\operatorname{rank}\left(S_{X}\right)$ & $S_{X}$ \\
\hline 19 & $U \oplus E_{8} \oplus E_{8} \oplus A_{1}$ \\
\hline 18 & $U \oplus E_{8} \oplus E_{8}$ \\
\hline 17 & $U \oplus E_{8} \oplus E_{7}$ \\
\hline 16 & $U \oplus E_{8} \oplus D_{6}$ \\
\hline 15 & $U \oplus E_{8} \oplus D_{4} \oplus A_{1}$ \\
\hline 14 & $U \oplus E_{8} \oplus D_{4}, U \oplus D_{8} \oplus D_{4}, U \oplus E_{8} \oplus A_{1}^{4}$ \\
\hline 13 & $U \oplus E_{8} \oplus A_{1}^{3}, U \oplus E_{7} \oplus A_{1}^{4}$ \\
\hline 12 & $U \oplus E_{8} \oplus A_{1}^{2}, U \oplus E_{7} \oplus A_{1}^{3}, U \oplus D_{6} \oplus A_{1}^{4}$ \\
\hline 11 & $U \oplus E_{8} \oplus A_{1}, U \oplus E_{7} \oplus A_{1}^{2}, U \oplus D_{6} \oplus A_{1}^{3}, U \oplus D_{4} \oplus A_{1}^{5}$ \\
\hline 10 & $U \oplus E_{8}, U \oplus D_{8}, U \oplus D_{4} \oplus D_{4}, U(2) \oplus D_{4} \oplus D_{4}$, \\
\hline 9 & $U \oplus E_{7} \oplus A_{1}, U \oplus D_{6} \oplus A_{1}^{2}, U \oplus D_{4} \oplus A_{1}^{4}, U \oplus A_{1}^{8}$ \\
\hline
\end{tabular}

Table $3 \quad\left(S_{X}\right.$ is not 2-elementary and $\left.\operatorname{rank}\left(S_{X}\right) \geq 9\right)$.

\begin{tabular}{c|c}
\hline $\operatorname{rank}\left(S_{X}\right)$ & $S_{X}$ \\
\hline 13 & $U \oplus E_{8} \oplus A_{3}$ \\
\hline 12 & $U \oplus E_{8} \oplus A_{2}$ \\
\hline 11 & $U \oplus E_{6} \oplus A_{2}$ \\
\hline 9 & $U \oplus A_{7}, U \oplus D_{4} \oplus A_{3}, U \oplus D_{5} \oplus A_{2}, U \oplus D_{7}, U \oplus E_{6} \oplus A_{1}$ \\
\hline
\end{tabular}




\section{$\S 2$. Finite automorphisms of $K 3$ surfaces}

Let $X$ be an algebraic $K 3$ surface. We denote by Aut $(X)$ the group of automorphisms of $X$. Let $G$ be a finite subgroup of $\operatorname{Aut}(X)$ and let $\omega_{X}$ be a nowhere vanishing holomorphic 2 -form on $X$. Then for $g \in G$, $g^{*} \omega_{X}=\alpha_{X}(g) \cdot \omega_{X}$ where $\alpha_{X}(g) \in C^{*}$. Therefore we have an exact sequence

$$
1 \longrightarrow K \longrightarrow G \stackrel{\alpha_{x}}{\longrightarrow} Z / m \longrightarrow 1
$$

where $Z / m$ is a cyclic group of $m$-th root of unity in $C^{*}$ and $K$ is the kernel of $\alpha_{X}$. Moreover the representation of the cyclic group $Z / m$ in $T_{X} \otimes \boldsymbol{Q}$ is isomorphic to a direct sum of irreducible representations of the cyclic group $\boldsymbol{Z} / m$ over $\boldsymbol{Q}$ of maximal rank $\phi(m)$, where $\phi$ is the Euler function. In particular $\phi(m) \leqq \operatorname{rank}\left(T_{X}\right)$ and hence $m \leq 66$ ([6], Theorem 3.1 and Corollary 3.2).

An automorphism $g$ of $X$ is called symplectic if $\alpha_{X}(g)=1$. The classification of finite symplectic automorphism groups of $K 3$ surfaces is recently given by S. Mukai [5], based on the study of abelian groups due to Nikulin [6].

Proposition 2.1 ([6], §5, [5], (0.1)). Let $g$ be a symplectic automorphism of finite order $n$ of a K3 surface. Then $n \leq 8$ and the number of fixed points $f(n)$ depends only on $n$ and is as follows:

\begin{tabular}{c|c|c|c|c|c|c|c}
\hline$n$ & 2 & 3 & 4 & 5 & 6 & 7 & 8 \\
\hline$f(n)$ & 8 & 6 & 4 & 4 & 2 & 3 & 2 \\
\hline
\end{tabular}

Let $G$ be a finite symplectic automorphism group of a $K 3$ surface. Put $f(1)=24$ and $\mu(G)=(1 /|G|) \sum_{g \in G} f(|g|)$. By the Lefschetz fixed point formula and an elementary representation theory, we have

Proposition 2.2 ([5], Proposition 2.4). $\mu(G)=2+\operatorname{rank}\left(L^{G}\right)$ where $L=H^{2}(X, Z)$ and $L^{G}=\left\{x \in L \mid g^{*} x=x\right.$ for any $\left.g \in G\right\}$.

In what follows we assume that $\operatorname{Aut}(X)$ is finite. Then we have an exact sequence

$$
1 \longrightarrow G_{X} \longrightarrow \operatorname{Aut}(X) \stackrel{\alpha_{X}}{\longrightarrow} Z / m \longrightarrow 1
$$

where $G_{X}$ is the kernel of $\alpha_{X}$. In Section 5 we shall need the following: 
LemmA 2.3. (i) If $\operatorname{rank}\left(S_{X}\right) \leq 14$, then $G_{X}$ is a subgroup of $Z / 3$ or $Z / 2 \times Z / 2$; (ii) If $\operatorname{rank}\left(S_{X}\right) \leq 12$, then $G_{X}$ is a subgroup of $Z / 2$; (iii) If $\operatorname{rank}\left(S_{X}\right) \leq 8$, then $G_{X}=\{1\}$.

Proof. It follows from [6], Theorem 1.1 that $L^{G_{X}}$ contains $T_{X}$. Since $G_{X}$ is finite, the signature of $S_{X}^{G_{X}}$ is equal to $(1, r)$, where $r$ is a non negative integer. Hence $\operatorname{rank}\left(L^{G_{X}}\right) \geq \operatorname{rank}\left(T_{X}\right)+1$. Note that $\operatorname{rank}\left(T_{X}\right)$ $+\operatorname{rank}\left(S_{X}\right)=22$. Now the assersions easily follows from Propositions 2.1 and 2.2 .

Proposition 2.4 ([6], § 10). Assume that $G=G_{X}$ is a subgroup of $Z / 3$ or $Z / 2 \times Z / 2$. Then the discriminant group $A_{L^{G}}$ of $L^{G}$ is as follows:

\begin{tabular}{c|c|c|c}
\hline$G$ & $Z / 2$ & $Z / 2 \times Z / 2$ & $Z / 3$ \\
\hline$A_{L^{a}}$ & $(Z / 2)^{8}$ & $(Z / 2)^{8} \times(Z / 4)^{2}$ & $(Z / 3)^{6}$ \\
\hline
\end{tabular}

\section{$\S 3$. Elliptic pencils on $K 3$ surfaces}

Let $X$ be a $K 3$ surface. An elliptic pencil $\pi: X \rightarrow P^{1}$ is a holomorphic map $\pi$ from $X$ to $\boldsymbol{P}^{1}$ whose general fibres are smooth elliptic curves. An effective divisor $D$ is called a $m$-section of $\pi$ if $D \cdot F=m$, where $F$ is a fibre of $\pi$ and $m \in N$. A 1-section is simply called a section. All singular fibres of an elliptic pencil were classified by Kodaira [1]. We use the terminology of singular fibres in [1]. The following lemma follows from [11], §3, Corollary 3, the Riemann-Roch theorem and the classification of singular fibres of elliptic pencils [1].

Lemma 3.1. Let $X$ be an algebraic $K 3$ surface and let $S_{X}$ be the Picard lattice of $X . \quad$ Assume that $S_{X}=U \oplus K$, where $K$ is a negative definite lattice. Then

(i) there exists an elliptic pencil $\pi: X \rightarrow \boldsymbol{P}^{1}$ with a section.

(ii) If $K=K_{1} \oplus N$, where $K_{1}$ and $N$ are negative definite lattices and $N$ is generated by elements with square $(-2)$, then $\pi$ has a singular fibre $F$ as in the following table:

\begin{tabular}{c|c|c|c|c|c|c|c}
\hline$N$ & $A_{1}$ & $A_{2}$ & $A_{n}(n \geq 3)$ & $D_{n}(n \geq 4)$ & $E_{6}$ & $E_{7}$ & $E_{8}$ \\
\hline$F$ & $\mathrm{I}_{2}$ or III & $\mathrm{I}_{3}$ or IV & $\mathrm{I}_{n+1}$ & $\mathrm{I}_{n-4}^{*}$ & $\mathrm{IV}^{*}$ & $\mathrm{III}^{*}$ & $\mathrm{II}^{*}$ \\
\hline
\end{tabular}


The following will be used in the latter to prove the existence of symplectic automorphisms.

Proposition 3.2 ([1], Theorem 12.2, [12], Corollaries 1.5, 1.7). Let $X$ be an algebraic $K 3$ surface and $S_{X}$ the Picard lattice of $X$. Let $\pi: X \rightarrow \boldsymbol{P}^{1}$ be an elliptic pencil with a section and let $F_{\nu}(1 \leq \nu \leq k)$ be all singular fibres of $\pi$. We denote respectively by $\varepsilon_{\nu}, m_{\nu}$ or $\mu_{\nu}$ the Euler number of $F_{\nu}$, the number of irreducible components of $F_{\nu}$ or the number of simple components of $F_{\nu}$. Then

(i) $\sum_{\nu=1}^{k} \varepsilon_{\nu}=24$ (= the Euler number of $\left.X\right)$,

(ii) $\operatorname{rank}\left(S_{X}\right)=r+2+\sum_{\nu=1}^{k}\left(m_{\nu}-1\right)$

where $r$ is the rank of the group of sections of $\pi$,

(iii) when $r=0$, let $n$ denote the order of the group of sections of $\pi$. Then we have

$$
\left|\operatorname{det}\left(S_{X}\right)\right|=\prod_{\nu=1}^{k} \mu_{\nu} / n^{2}
$$

\section{§4. Proof of the Theorem-the case when $\operatorname{rank}\left(S_{X}\right) \geq 15$}

In this section and the next we prove our theorem. By our proof in the following, we can see:

Proposition. Let $X$ be an algebraic $K 3$ surface with finite automorphism group Aut $(X)$. Then the subgroup $G_{X}$ of symplectic automorphisms of $\operatorname{Aut}(X)$ is uniquely determined by the isomorphism class of $S_{X}$.

The assersion (ii) in Theorem follows from this Proposition and the exact sequence (1). For simplicity, in the following, we assume that $X$ is a general algebraic $K 3$ surface with finite automorphism group.

Let $X$ be a general algebraic $K 3$ surface with finite automorphism group and $\operatorname{rank}\left(S_{X}\right) \geq 15$. Then $S_{X}$ is a 2-elementary lattice (see Table 2). By [8], Section 4, there exists an automorphism $\sigma$ of order 2 such that $\sigma^{*} \mid S_{X}=1_{S_{X}}$ and $\sigma^{*} \mid T_{X}=-1_{T_{X}}$. Therefore we have an exact sequence:

$$
1 \longrightarrow G_{X} \longrightarrow \operatorname{Aut}(X) \stackrel{\alpha_{X}}{\longrightarrow} Z / 2 \longrightarrow 1
$$

where $Z / 2$ is generated by $\sigma$. Since $g^{*} \mid T_{X}=1_{T_{X}}$ for all $g \in G_{X}, g^{*} \circ \sigma^{*}=$ $\sigma^{*} \circ g^{*}$. It follows from the global Torelli theorem [11] that $g \circ \sigma=\sigma \circ g$. Hence the above exact sequence splits: $\operatorname{Aut}(X) \simeq G_{X} \times Z / 2$.

A dual graph of smooth rational curves is the following simplicial complex $\Gamma$ : (i) the set of vertices is a set of smooth rational curves on 
$X$; (ii) the vertices $C, C^{\prime}$ are joined by $m$-tuple line if $C \cdot C^{\prime}=m$.

To determine the group $G_{X}$ we use the dual graph of all smooth rational curves on $X$. Such graphs were found by Nikulin [8]. However for $S_{X}=U \oplus E_{8} \oplus E_{8} \oplus A_{1}$, his graph is not complete (compare the following graph in Figure 1 with the table 2 in [8], $\S 4$, Part 5). It follows from [13], Proposition 1 and [14], Lemma 2.4 that the following graph represents all smooth rational curves on $X$.

Let $\Gamma$ be the dual graph of all smooth rational curves on $X$ (see Figures 1-5). Consider the natural homomorphism $\rho: \operatorname{Aut}(X) \rightarrow \operatorname{Aut}(\Gamma)$, where $\operatorname{Aut}(\Gamma)$ is the symmetry group of $\Gamma$. Since $S_{X}$ is generated by the classes of smooth rational curves in $\Gamma$, the kernel of $\rho$ acts on $S_{X}$ as identity. Hence the symplectic group $G_{X}$ is regarded as a subgroup of $\operatorname{Aut}(T)$.

(4.1) $S_{X}=U \oplus E_{8} \oplus E_{8} \oplus A_{1}$. The following diagram $\Gamma$ is the dual graph of all smooth rational curves on $X$ :

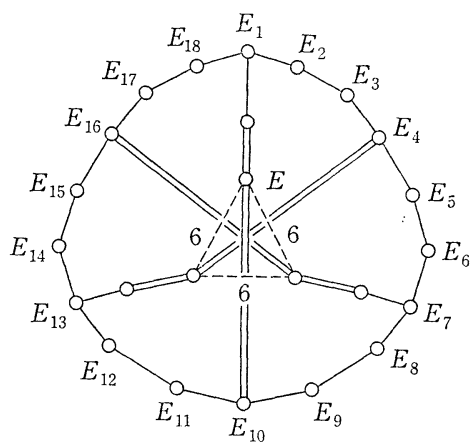

Figure 1

Obviously the symmetry group $\operatorname{Aut}(\Gamma)$ is isomorphic to $\mathfrak{S}_{3}$.

We now claim that $G_{X} \simeq \widetilde{S}_{3}$. First consider the elliptic pencil $\left|\Delta_{1}\right|=$ $\left|\sum_{i=1}^{18} E_{i}\right|$ which has a section and a singular fibre of type $\mathrm{I}_{18}$. By the formulas in Proposition 3.2, we can see that $\left|\Delta_{1}\right|$ has only one reducible singular fibre of type $I_{18}$ and the group of sections of $\left|\Delta_{1}\right|$ is isomorphic to $Z / 3$. These sections act on $X$ as a symplectic automorphism of order 3 which is a rotation of $\Gamma$ of order 3 . Next consider the elliptic pencil $\left|\Delta_{2}\right|=\left|E+E_{10}\right|$ which has a section and two singular fibres of type $\mathrm{I}_{2}$ and of type $\mathrm{I}_{12}^{*}$. Again it follows from the formulas in Proposition 3.2 that $\left|\Delta_{2}\right|$ has only two reducible singular fibres of type $I_{2}$ and of type 
$I_{12}^{*}$ and the group of sections of $\left|\Delta_{2}\right|$ is isomorphic to $Z / 2$. Therefore $G_{X} \simeq \mathfrak{S}_{3}$.

(4.2) $S_{X}=U \oplus E_{8} \oplus E_{8}$. The following diagram $\Gamma$ is the dual graph of all smooth rational curves on $X$ :

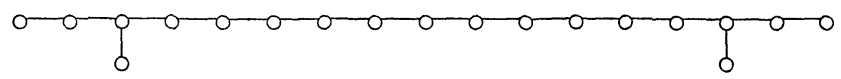

Figure 2

We claim that $G_{X} \simeq \operatorname{Aut}(\Gamma)(\simeq Z / 2)$. Let $\varphi$ be an isometry of $S_{X}$ defined by $\varphi((x, y, z))=(x, z, y)$ where $(x, y, z) \in U \oplus E_{8} \oplus E_{8}$. Note that the second cohomology lattice $L=H^{2}(X, Z)$ is the direct sum of $S_{X}$ and $T_{X}$. Put $\tilde{\varphi}=\left(\varphi, 1_{T_{X}}\right): S_{X} \oplus T_{X} \rightarrow S_{X} \oplus T_{X}$. Then by the global Torelli theorem [11], there exists an automorphism $g$ of $X$ such that $g^{*}=\tilde{\varphi}$ on $L$. By construction, $g$ is symplectic and generates Aut $(\Gamma)$. Hence $G_{X} \simeq Z / 2$ and $\operatorname{Aut}(X) \simeq Z / 2 \times Z / 2$.

(4.3) $S_{X}=U \oplus E_{8} \oplus E_{7}$. The following diagram $\Gamma$ is the dual graph of all smooth rational curves on $X$ :

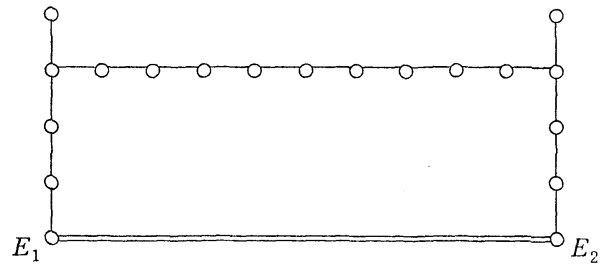

Figure 3

Obviously $\operatorname{Aut}(\Gamma) \simeq Z / 2$. By considering the elliptic pencil $\left|E_{1}+E_{2}\right|$ with a section, we have a symplectic automorphism of order 2 which acts on $\Gamma$ as a symmetry of order 2 . Hence we have $\operatorname{Aut}(X) \simeq Z / 2 \times Z / 2$.

(4.4) $S_{X}=U \oplus E_{8} \oplus D_{6}$. The following diagram $\Gamma$ is the dual graph of all smooth rational curves on $X$ :

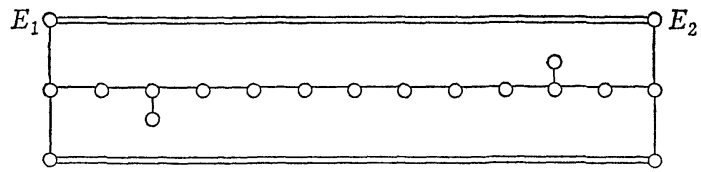

Figure 4 
We can see $\operatorname{Aut}(\Gamma) \simeq Z / 2 \times Z / 2$. We select a generator $\left\{\gamma_{1}, \gamma_{2}\right\}$ of $\operatorname{Aut}(\Gamma)$ as follows; $\gamma_{1}$ is the reflection of $\Gamma$ with $\gamma_{1}\left(E_{1}\right)=E_{2}$ and $\gamma_{2}$ is the reflection with respect to the middle horizontal line. By considering the elliptic pencil $\left|E_{1}+E_{2}\right|$ with a section, we have a symplectic automorphism $g$ whose action on $\Gamma$ coincides with $\gamma_{1}$. On the other hand, if $\gamma_{2}$ is represented by a symplectic automorphism $g^{\prime}$, then $g^{\prime}$ preserves 15 smooth rational curves respectively (see Figure 4). Hence the number of fixed points of $g^{\prime}$ is greater than 8 which is impossible (Proposition 2.1). Thus we have $G_{X} \simeq Z / 2$ and $\operatorname{Aut}(X) \simeq Z / 2 \times Z / 2$.

(4.5) $S_{X}=U \oplus E_{8} \oplus D_{4} \oplus A_{1}$. The following diagram $\Gamma$ is the dual graph of all smooth rational curves on $X$ :

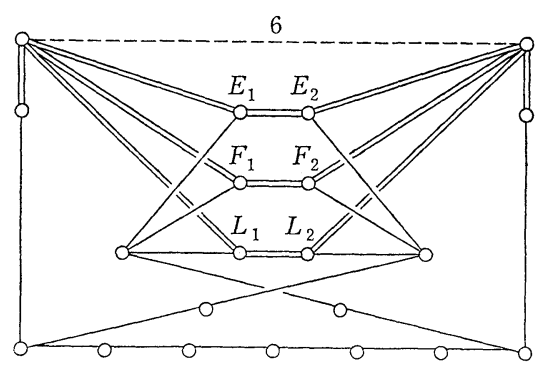

Figure 5

We can see that $\operatorname{Aut}(\Gamma) \simeq \mathfrak{S}_{3} \times \boldsymbol{Z} / 2$ where $Z / 2$ is generated by the reflection $\gamma$ with $\gamma\left(E_{1}\right)=E_{2}$ and $\widetilde{S}_{3}$ is the permutations of the set $\left\{E_{1}, F_{1}, L_{1}\right\}$. By considering the elliptic pencil $\left|E_{1}+E_{2}\right|$ with a section, $\gamma$ is represented by a symplectic automorphism of order 2 . On the other hand, any element of $\widetilde{S}_{3}$ is not represented by a symplectic automorphism because a symplectic automorphism of order 2 (resp. of order 3 ) has exactly 8 (resp. 6) isolated fixed points (Proposition 2.1). Therefore we have $G_{X} \simeq Z / 2$ and $\operatorname{Aut}(X) \simeq Z / 2 \times Z / 2$.

\section{§5. Proof of the Theorem-the case when $\operatorname{rank}\left(S_{X}\right) \leq 14$}

(5.1) First we remark that $G_{X}$ is trivial if $\operatorname{rank}\left(S_{X}\right) \leq 8$ (Lemma 2.3, (iii)). Hence it suffices to consider the case that $9 \leq \operatorname{rank}\left(S_{X}\right) \leq 14$. In these cases, $G_{X}$ is a subgroup of $Z / 2 \times Z / 2$ or $Z / 3$ (Lemma 2.3). Consider a primitive embedding $T_{X} \subset L^{G_{X}}$ and denote by $T_{\bar{X}}^{\perp}$ the orthogonal com. plement of $T_{X}$ in $L^{G_{X}}$. Then $T_{X} \oplus T_{\bar{X}}^{\frac{1}{X}}$ is a sublattice of $L^{G_{X}}$ of finite index and $A_{L^{a_{X}}}$ is a quotient group of $A_{T_{X} \oplus T^{\frac{1}{X}}}$, and hence $l\left(T_{X} \oplus T_{\bar{X}}^{\perp}\right) \geq l\left(L^{G_{X}}\right)$. 
Since $\operatorname{rank}\left(T_{\bar{X}}^{\perp}\right) \geq l\left(T_{\bar{X}}^{\perp}\right)$ and $l\left(T_{X}\right)=l\left(S_{X}\right)$, we have $l\left(S_{X}\right)+\operatorname{rank}\left(T_{\frac{1}{X}}\right) \geq$ $l\left(L^{a_{X}}\right)$. Therefore it follows from Proposition 2.4 that:

$G_{X}=\{1\}$ or $Z / 2$ if $S_{X}=U \oplus E_{8} \oplus D_{4}, U \oplus D_{8} \oplus D_{4}, U \oplus E_{8} \oplus A_{1}^{4}, U \oplus E_{7}$ $\oplus A_{1}^{4}, \quad U \oplus D_{6} \oplus A_{1}^{4}, \quad U \oplus D_{4} \oplus A_{1}^{6}, \quad U \oplus D_{4} \oplus A_{1}^{5}, \quad U \oplus E_{8} \oplus A_{1}^{3}, \quad U \oplus E_{7} \oplus A_{1}^{3}, \quad U(2)$ $\oplus D_{4} \oplus D_{4}, U \oplus D_{6} \oplus A_{1}^{3}, U \oplus A_{1}^{7}, U \oplus D_{4} \oplus A_{1}^{4}, U \oplus A_{1}^{8}$ or $U(2) \oplus A_{1}^{7}$ and $G_{X}=$ $\{1\}$ if $S_{X}$ is otherwise. Moreover, if $G_{X}=Z / 2$ and $S_{X}=U \oplus E_{8} \oplus A_{1}^{3}, U \oplus$ $E_{7} \oplus A_{1}^{3}, U \oplus D_{6} \oplus A_{1}^{3}, U \oplus A_{1}^{7}$ or $U \oplus D_{4} \oplus A_{1}^{4}$, then $A_{L^{\sigma_{X}}}=A_{T \oplus T_{\bar{X}}}$ and hence $L_{X}^{G_{X}}=T_{X} \oplus T_{\frac{1}{\bar{X}}}$. This is a contradiction because $L_{X}^{G_{X}}$ is a 2-elementary lattice with $\delta_{L^{\sigma_{X}}}=0$ and, on the other hand, $T_{X}$ is a 2-elementary lattice with $\delta_{T_{X}}=1$. Also, if $S_{X}=U \oplus E_{8} \oplus D_{4}$ and $G_{X}=Z / 2$, then $l\left(L^{G_{X}}\right)=l\left(T_{X}\right)$ $+l\left(T_{\frac{1}{X}}\right)$ and hence $L^{G_{X}}=T_{X} \oplus T_{\bar{X}}$. Hence $T_{\frac{1}{X}}$ is a 2-elementary lattice with $\operatorname{rank}\left(T_{\frac{1}{X}}\right)=6, l\left(T_{\frac{1}{X}}\right)=6$ and $\delta_{T_{\frac{1}{X}}}=0$. However, by [7], Theorem 3.6.2, such lattice does not exist.

Hence $G_{X}=\{1\}$ if $S_{X}=U \oplus E_{8} \oplus D_{4}, U \oplus E_{8} \oplus A_{1}^{3}, U \oplus E_{7} \oplus A_{1}^{3}, U \oplus D_{6}$ $\oplus A_{1}^{3}, U \oplus D_{4} \oplus A_{1}^{4}$ or $U \oplus A_{1}^{7}$.

In the following we shall see that $G_{X}=Z / 2$ if $S_{X}=U \oplus D_{8} \oplus D_{4}$, $U \oplus E_{8} \oplus A_{1}^{4}, U \oplus E_{7} \oplus A_{1}^{4}, U \oplus D_{6} \oplus A_{1}^{4}, U \oplus D_{4} \oplus A_{1}^{6}, U \oplus D_{4} \oplus A_{1}^{5}, U(2) \oplus D_{4} \oplus$ $D_{4}, U \oplus A_{1}^{8}$ or $U(2) \oplus A_{1}^{7}$.

(5.2) $\quad S_{X}=U \oplus D_{8} \oplus D_{4}$. Note that there exists an elliptic pencil with a section whose reducible singular fibres are of type $\mathrm{I}_{0}^{*}$ and of type $\mathrm{I}_{4}^{*}$ (Lemma 3.1). Hence we have the following dual graph of smooth rational curves on $X$ :

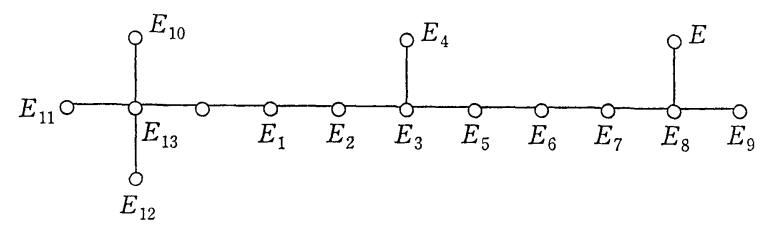

where $E_{1}$ is a section of this pencil and others are components of singular fibres. Let us consider the elliptic pencil $|\Delta|=\mid 2 E_{1}+4 E_{2}+6 E_{3}+3 E_{4}+$ $5 E_{5}+4 E_{6}+3 E_{7}+2 E_{8}+E_{9}$. Then $E_{10}, E_{11}, E_{12}$ and $E_{13}$ are components of a singular fibre $F$ of this pencil $|4|$. By Proposition 3.2, $F$ is of type $I_{0}^{*}$ and hence there exists a smooth rational curve $E_{14}$ with $E_{10}+E_{11}+E_{12}$ $+E_{14}+2 E_{13} \in|\Delta|$. Since $E$ is a 2 -section of $|\Delta|, E \cdot E_{14}=2$. Then the elliptic pencil $\left|E_{14}+E\right|$ has two sections $E_{13}, E_{8}$ and these two sections define a symplectic automorphism. Therefore $G_{X} \simeq Z / 2$.

(5.3) $\quad S_{X}=U \oplus E_{8} \oplus A_{1}^{4}$. First we remark that $U \oplus E_{8} \oplus A_{1}^{4}$ is isomor- 
phic to $U \oplus E_{7} \oplus D_{4} \oplus A_{1}$ (Proposition 1.1). Therefore there exists an elliptic pencil with a section which has three reducible singular fibres of type III*, $I_{0}^{*}$ and $I_{2}$ (Lemma 3.1). Hence we have the following dual graph of smooth rational curves on $X$ :

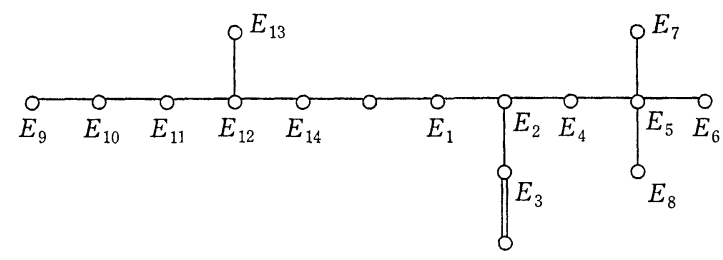

where $E_{2}$ is a section of this pencil and others are components of singular fibres. Consider the elliptic pencil $|\Delta|=\mid E_{1}+E_{3}+E_{6}+E_{7}+2\left(E_{2}+E_{4}\right.$ $\left.E_{5}\right) \mid$. Then $E_{j}, 9 \leq j \leq 14$, are contained in some singular fibre $F$ fo $|\Delta|$. It follows from Proposition 3.2 that $F$ is of type $I_{2}^{*}$. Hence there exists a smooth rational curve $E$ with $E+E_{9}+E_{13}+E_{14}+2\left(E_{10}+E_{11}+E_{12}\right) \in|\Delta|$. Since $E_{8}$ is a 2-section of $|\Delta|, E \cdot E_{8}=2$. Then the elliptic pencil $\left|E+E_{8}\right|$ has two sections $E_{5}$ and $E_{10}$ which define a symplectic automorphism of order 2. Therefore we have $G_{X} \simeq Z / 2$.

(5.4) $\quad S_{X}=U \oplus E_{7} \oplus A_{1}^{4} . \quad$ First note that $U \oplus E_{7} \oplus A_{1}^{4} \simeq U \oplus D_{6} \oplus D_{4} \oplus A_{1}$ (Proposition 1.1). Since there exists an elliptic pencil with a section which has three reducible singular fibres of type $I_{2}^{*}, I_{0}^{*}$ and $I_{2}$ (Lemma 3.1), we have the following dual graph:

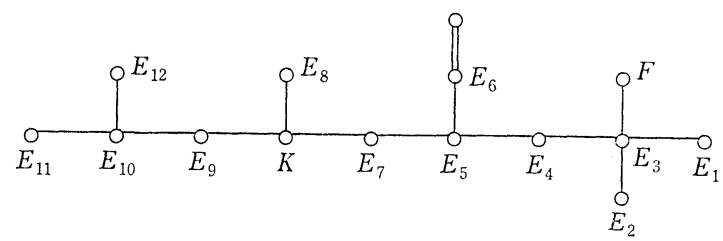

where $E_{5}$ is a section of this pencil and others are components of singular fibres. Consider the elliptic pencil $|\Delta|=\mid E_{1}+E_{2}+E_{6}+E_{7}+2\left(E_{3}+E_{4}\right.$ $\left.+E_{5}\right)$. Then $E_{j}, 8 \leq j \leq 12$, are components of singular fibres of $|\Delta|$. Since $K$ is a section of $|\Delta|$ and $K \cdot E_{8}=K \cdot E_{9}=1, E_{8}$ is not a component of a singular fibre containing $E_{9}$. It now follows from Proposition 3.2 that the reducible singular fibres of $|\Delta|$ are of type $I_{2}^{*}, I_{0}^{*}$ and $I_{2}$. Hence there exists a smooth rational curve $E$ with $E+E_{9}+E_{11}+E_{12}+2 E_{10} \epsilon$ $|\Delta|$. Since $F$ is a 2 -section of $|\Delta|, E \cdot F=2$. The elliptic pencil $|E+F|$ has two sections $E_{3}$ and $E_{10}$, and hence $G_{X} \simeq Z / 2$. 
(5.5) $\quad S_{X}=U \oplus D_{6} \oplus A_{1}^{4} . \quad$ First note that $U \oplus D_{6} \oplus A_{1}^{4} \simeq U \oplus D_{4} \oplus D_{4} \oplus A_{1}^{2}$ (Proposition 1.1). Since there exists an elliptic pencil with a section which has 4 reducible singular fibres of type $\mathrm{I}_{0}^{*}, \mathrm{I}_{0}^{*}, \mathrm{I}_{2}$ and $\mathrm{I}_{2}$ (Lemma 3.1), we have the following dual graph:

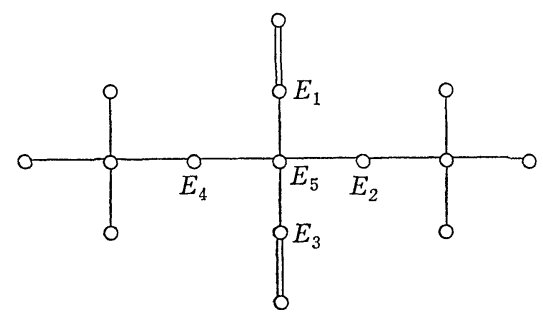

where $E_{5}$ is a section of this pencil and others are components of singular fibres. Then the elliptic pencil $\left|E_{1}+E_{2}+E_{3}+E_{4}+2 E_{5}\right|$ has two sections. Hence $G_{X} \simeq Z / 2$.

(5.6) $S_{X}=U \oplus D_{4} \oplus A_{1}^{6}$. Since there exists an elliptic pencil with a section which has one singular fibre of type $\mathrm{I}_{0}^{*}$ and 6 singular fibres of type $I_{2}$ (Lemma 3.1), we have the following dual graph of smooth rational curves:

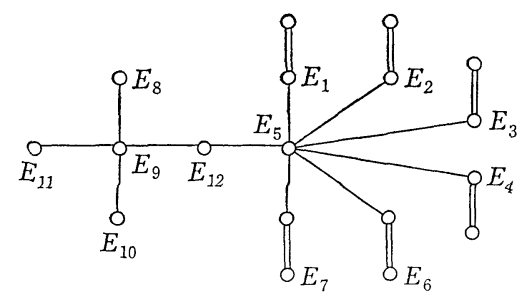

where $E_{5}$ is a section of this pencil and others are components of singular fibres. Consider the elliptic pencil $|\Delta|=\left|E_{1}+E_{2}+E_{3}+E_{4}+2 E_{5}\right|$. Then $E_{j}, 6 \leq j \leq 11$, are components of singular fibres of $|\Delta|$. By Proposition 3.2, the following two cases occur: $(\alpha)|\Delta|$ has reducible singular fibres of type $I_{0}^{*}, I_{0}^{*}, I_{2}$ and $I_{2} ;(\beta)|\Delta|$ has two reducible singular fibres of type $I_{2}^{*}$ and $\mathrm{I}_{0}^{*}$. In case $(\alpha)$, we may assume that there exists a smooth rational curve $E$ with $E+E_{6} \in\left\{\Delta \mid\right.$. Since $E_{12}$ is a 2-section of $\{\Delta\}$, we have $E \cdot E_{12}$ $=2$. Then the elliptic pencil $\left|E+E_{12}\right|$ has two sections $E_{5}$ and $E_{9}$, and hence $G_{X} \simeq Z / 2$. In case ( $\beta$ ), we may assume that there exists a smooth rational curve $F$ with $E_{6}+E_{7}+E_{8}+E_{11}+2 E_{9}+2 E_{10}+2 F \in|\Delta|$. Then the elliptic pencil $\left|E_{8}+E_{10}+E_{11}+E_{12}+2 E_{9}\right|$ has two sections $E_{5}$ and $F$, and hence $G_{X} \simeq Z / 2$. 
(5.7) $S_{X}=U \oplus D_{4} \oplus A_{1}^{5}$. In this case, the same argument as in (5.6) shows $G_{X} \simeq Z / 2$.

(5.8) $\quad S_{X}=U(2) \oplus D_{4} \oplus D_{4}$. First we claim that $S_{X}$ is isomorphic to $U \oplus K$, where $K$ is a negative definite lattice of rank 8 . Let $\{e, f\}$ be a basis of $U(2)$ and $\left\{e_{j}\right\},\left\{f_{j}\right\}$ the two copies of a basis of $D_{4}$ as in the following dual graphs:
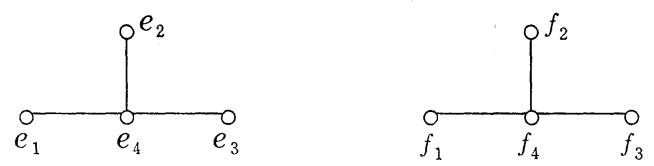

Put $\delta=e+f+e_{1}+f_{1}$. Then $\delta^{2}=0$ and $\left\langle\delta, e_{4}\right\rangle=1$. Hence $\delta$ and $e_{4}$ generate a sublattice of $S_{X}$ isomorphic to $U$. So we have $S_{X} \simeq U \oplus K$. Therefore there exists an elliptic pencil $|\Delta|$ with a section (Lemma 3.1). It follows from Proposition 3.2, (ii) that $K$ has a sublattice $K^{\prime}$ of finite index which is generated by some components of singular fibres of $|\Delta|$. Since $K$ is a 2-elementary lattice with rank $K=8$, det $K=2^{6}$ and $\delta_{K}=0$, we can see that $K \neq K^{\prime}$. Hence the group of section of $|\Delta|$ is not trivial (Proposition 3.2, (iii)). Therefore $G_{X} \simeq Z / 2$.

(5.9) $S_{X}=U \oplus A_{1}^{8}, U(2) \oplus A_{1}^{7}$. In these cases, to prove $G_{X} \simeq Z / 2$, we give a lattice theoretic construction of a symplectic automorphism.

In case $S_{X}=U \oplus A_{1}^{8}$, consider a sublattice $\langle 2\rangle \oplus\langle-2\rangle \oplus A_{1}^{8}$ of $S_{X}$. Since a 2-elementary lattices $S$ is determined by $\operatorname{rank}\left(S_{X}\right), l(S)$ and the parity of $S$, this sublattice is isomorphic to $\langle 2\rangle \oplus\langle-2\rangle \oplus E_{8}(2)$ (Proposition 1.1). By this isomorphism, we consider $\langle 2\rangle \oplus\langle-2\rangle \oplus E_{8}(2)$ as a sublattice of $S_{X}$. Let $\iota$ be an involution of $\langle 2\rangle \oplus\langle-2\rangle \oplus E_{8}(2)$ such that $\iota \mid\langle 2\rangle \oplus\langle-2\rangle$ $=1$ and $\iota \mid E_{8}(2)=-1$. Since $\langle 2\rangle \oplus\langle-2\rangle$ and $E_{8}(2)$ are 2-elementary, $\iota$ extends to an involution $\iota^{\prime}$ of $S_{X}$. By construction, $\iota^{\prime}$ acts on the discriminant group $A_{S_{X}}$ as identity. Hence $\iota^{\prime}$ extends to an involution $\tilde{\iota}$ of $L_{X}$ with $\tilde{\iota} \mid T_{X}=1$. $\tilde{\iota}$ preserves a period of $X$ and the Kähler cone because $E_{8}(2)$ contains no (-2)-elements. Hence by the global Torelli theorem [11], $\iota$ is represented by a symplectic automorphism of order 2 .

In case $S_{X}=U(2) \oplus A_{1}^{7}$, we define two involutions $\sigma$ and $g$ of $L_{X}$ as follows: let $\left\{\alpha_{i}, \beta_{i}\right\}$ be a copy of a basis of $U(1 \leq i \leq 3)$ and let $\left\{e_{j}\right\},\left\{f_{j}\right\}$ be copies of a basis of $E_{8}(1 \leq j \leq 8)$. Then $\left\{\alpha_{i}, \beta_{i}, e_{j}, f_{j} \mid 1 \leq i \leq 3,1 \leq j\right.$ $\leq 8\}$ is a basis of $L_{X}=U \oplus U \oplus U \oplus E_{8} \oplus E_{8}$. Put $g \mid U \oplus U \oplus U=1$ and 
$g\left(e_{j}\right)=f_{j}, \quad 1 \leq j \leq 8, \sigma\left(\alpha_{1}\right)=\beta_{1}, \sigma\left(\alpha_{i}\right)=-\alpha_{i}, \sigma\left(\beta_{i}\right)=-\beta_{i}, 2 \leq i \leq 3$, and $\sigma\left(e_{j}\right)=-f_{j}, 1 \leq j \leq 8$. Then $L^{\langle\sigma\rangle}$ is isomorphic to $\langle 2\rangle \oplus E_{8}(2) \simeq U(2) \oplus A_{1}^{7}$ which is generated by $\left\{\alpha_{1}+\beta_{1}, e_{j}-f_{j} \mid j=1, \cdots, 8\right\}$. On the other hand $L^{\langle g\rangle}$ is isomorphic to $U \oplus U \oplus U \oplus E_{8}(2)$ which is generated by $\left\{\alpha_{i}, \beta_{i}, e_{j}+\right.$ $\left.f_{j} \mid i=1,2,3, j=1, \cdots, 8\right\}$. How we consider $L_{X}^{\langle\sigma\rangle}$ as a Picard lattices $S_{X}$. Then we can easily see that $g$ preserves the Kähler cone of $X$ and a period of $X$. Hence by the global Torelli theorem [11], $g$ is represented by a symplectic automorphism. Thus we have $G_{X} \simeq Z / 2$.

\section{REFERENCES}

[1] Kodaira, K., On compact analytic surfaces II, Ann. Math., 77 (1963), 563-626. III, Ann. Math., 78 (1963), 1-40.

[2] Kondō, S., Enriques surfaces with finite automorphism groups, Japanese J. Math. (New Series), 12, (1986), 191-282.

[ 3 ] — On algebraic $K 3$ surfaces with finite automorphism groups, Proc. Japan Acad., 62, Ser. A, No. 9 (1986), 353-355.

[4] - - On automorphisms of algebraic $K 3$ surfaces which acts trivially on Picard groups, Proc. Japan Acad., 62, Ser. A, No. 9 (1986), 356-359.

[5] Mukai, S., Finite groups of automorphisms of $K 3$ surfaces and the Mathieu group, Invent. math., 94 (1988), 183-221.

[6] Nikulin, V. V., Finite automorphism groups of Kähler surfaces of type K3, Proc. Moscow Math. Soc., 38 (1979), 75-137.

[7] - Integral symmetric bilinear forms and some of their applications, Math. USSR Izv., 14 (1980), 103-167.

[8] - On the quotient groups of the automorphism groups of hyperbolic forms by the subgroups generated by 2-reflections, J. Soviet Math., 22 (1983), 1401-1476.

[9] - On a description of the automorphism groups of Enriques surfaces, Soviet Math. Dokl., 30 (1984), 282-285.

[10] - Surfaces of type $K 3$ with a finite automorphism group and a Picard group of rank three, Proc. Steklov Institute of Math. Issue, 3 (1985), 131-155.

[11] Piatetskii-Shapiro, I., Shafarevich, I. R., A Torelli theorem for algebraic surfaces of type K3, Math. USSR Izv., 35 (1971), 530-572.

[12] Shioda, T., On elliptic modular surfaces, J. Math. Soc. Japan, 24 (1972), 20-59.

[13] Vinberg, E. B., On groups of unit elements of certain quadratic forms, Math. USSR Sbornik, 16 (1972), 17-35.

[14] — Some arithmetic discrete groups in Lobachevskii spaces, in "Discrete subgroups of Lie groups and applications to Moduli", Tata-Oxford (1975), 323-348.

Department of Mathematical Sciences

Tokyo Denki University

Hatoyama-machi, Hiki-gun,

Saitama-ken, 350-03 Japan 